\title{
Aging and occupational and environmental medicine
}

In this issue of Occupational and Environmental Medicine we join other journals in the BMJ group and many other biomedical journals worldwide in publishing articles related to a global theme. The chosen theme-aging-was selected by means of a Delphi process among the editors of all the journals concerned. At around the end of October, all the participating journals will carry an issue devoted wholly or partially to papers on the theme of aging. Nine of the articles in this issue of Occupational and Environmental Medicine are about some aspect of aging.

What does age have to do with occupational and environmental medicine? As it turns out, quite a lot. Firstly, age can distort or modify associations between exposures and health effects. When carrying out epidemiological studies of the effects of occupational or environmental exposures on human health, age has to be taken into account as it is often a confounder or effect modifier. Inadequate or inappropriate controlling for age is a common -error. In an article in our Methodology series, Consonni and colleagues discuss why age should be taken into account and the techniques for doing it.

As people get older, they are at higher risk of several diseases and their resistance to harmful exposures is reduced. Musculoskeletal conditions are a good example. In two papers de Zwart and colleagues look at the effects of age and work demands or musculoskeletal complaints and the possibly distorting effects of workforce selection related to musculoskeletal complaints. And Nurminen revisits back pain in construction workers, taking into account physical work demands and aging. On the related topic of injuries at work, McNamee and coauthors discuss how the outcome of falls at work may be more serious among older women (in this case, older means more than 45 years old!).

The health effects of air pollution are topical at present. There is evidence that short term high levels (peaks) of some common city air pollutants are related to short term increases in morbidity and mortality from respiratory and cardiovascular diseases; but can exposure to higher levels of air pollution actually reduce life expectancy? Brunekreef's article reviews this still controversial area.

It is well known that the population, in developed countries at least, is aging. The workforce is also aging and there are concerns about what this might mean, as older workers have less physical capacity and stamina, sometimes reduced cognitive abilities, and may be more susceptible to workplace hazards than their younger colleagues. How should employers respond to the challenge of an aging workforce and what role can occupational health professionals play? We have three articles relating to these concerns.

The effects of aging on the circadian body clock are considered in the article by Reilly et al. Those of us whose work requires frequent travel across time zones or who work changing shifts know well that disturbances to the circadian body clock can have adverse personal health effects which can impinge on efficiency and productivity. Does the body clock behave differently in older people and do they tolerate disturbances less well than younger people? This could have important implications for work design with an aging workforce.

A viewpoint article by Snashall comes close to home and considers the healthcare profession and the difficulties this sort of exacting, stressful work can present for people as they get older. In some occupations that require stamina and a high level of skill (physical and mental), especially those where substandard practice would put other people at risk, repeated testing of capabilities and health status are mandatory and there are age limits for practice. Airline pilots are an example. Some people argue that certain categories of healthcare professionals-such as surgeons undertaking long, complex procedures-should be subject to similar requirements. This remains controversial and Snashall makes some suggestions that not everyone will agree with.

The final article in our cluster on the aging theme, by Westerholm and Kilbom, sets out the issues about age and work from the perspective of the occupational health professional.

The aging worker is here to stay. Rather than take a negative view of the problems this poses for employers, the advantages of older workers should be emphasised: they have a wealth of experience and accumulated skills, they are more likely to be stable and settled in their work as well as home life, and they are often loyal and committed employees. And it may well actually benefit the health of older people if they keep working, as work can provide mental and physical stimulation and a sense of self worth.

If an aging workforce means that attitudes and practices of employers have to change, so much the better. The attitude of some (one would like to think few) employers of "use them until they wear out and then get a new lot" is no longer tenable as the new lot (young, fit workers) will become thin on the ground. Taking the extra time and trouble to fit the job to the worker, rather than the worker to the job, can pay dividends for employers whether the workers are old or young.

So the messages are really quite simple. Age should be taken into account properly when undertaking occupational epidemiological studies; there are differences with age in how people respond to noxious exposures at work and in the general environment; body physiology changes with age in ways that are relevant to work requirements; older workers can and should be accommodated within the workplace; some specialised jobs cannot be made suitable for older workers but most can, with benefit to both workers and employers. While writing this editorial I have disturbed my body clock by travelling over several time zones and felt age creeping up on me; I am better able to cope with my work because of experience but less resilient to working days of 18 hours plus than I was 20 years ago. On the other hand, I love my work and would go mad if I had to stop, so I am certainly hoping to be one of those for whom work can be elegantly adapted as I grow older, allowing me to carry on into a ripe old age! 